\title{
Long-Term Outcome of a cohort of 272 patients undergoing sialendoscopy
}

\author{
Arike Vanden Daele ${ }^{1}$, Joekio Drubbel ${ }^{1}$, Charlotte Van Lierde ${ }^{1}$, Jeroen Meulemans ${ }^{1}$, \\ Pierre Delaere ${ }^{1}$, and Vincent Vander Poorten ${ }^{1}$ \\ ${ }^{1} \mathrm{KU}$ Leuven University Hospitals Leuven
}

July 22, 2021

\begin{abstract}
Objectives: To assess the cohort of patients undergoing sialendoscopic intervention for improvement of symptoms and glandrelated quality of life at long-term follow up. Design: retrospective review of medical records with a prospective follow-up by questionnaire. Methods: All patients undergoing sialendoscopy between March 2008 and June 2020 were analyzed in detail regarding indications, technical aspects and postoperative course. To maximize follow-up, patients also received a questionnaire by e-mail. Results: 272 sialendoscopies were performed in 221 patients. Median follow-up time was 37 months. The sialendoscopies were performed in 130 patients for lithiasis, in 66 for stenosis, in 14 for recurrent parotitis of childhood, in 8 for recurrent sialadenitis of unknown origin and in 3 for radio-iodine induced sialadenitis. Complications occurred in 11 of 272 sialendoscopies (4\%). Those were iatrogenic perforations, temporary lingual nerve paresthesia and swelling of the floor of the mouth. $53 \%$ of patients returned the questionnaire, for a total of 146 evaluable sialendoscopies. The majority of the responders indicated that sialendoscopy had improved their symptoms (83.6\%). Salivary glands could be preserved in $89 \%$ of the responder group. The highest percentage of patients reporting residual symptoms was found in the RPC group (81.3\%) and the lowest in the lithiasis group (16.2\%). Besides age, no statistical differences in demographic and pathological features between the responder and non-responder groups were found, supporting generalization of the responders' results to the entire cohort. Conclusions: This study confirms the good long-term outcomes of sialendoscopic interventions in patients with chronic sialadenitis of different etiologies and a high rate of gland preservation.
\end{abstract}

\section{$A B S T R A C T$}

Objectives: To assess the cohort of patients undergoing sialendoscopic intervention for improvement of symptoms and gland-related quality of life at long-term follow up.

Methods: This is a retrospective review of medical records with a prospective follow-up by questionnaire. All patients undergoing sialendoscopy between March 2008 and June 2020 were analyzed in detail regarding indications, technical aspects and postoperative course. To maximize follow-up, patients also received a questionnaire by e-mail.

Results: 272 sialendoscopies were performed in 221 patients. Median follow-up time was 37 months. The sialendoscopies were performed in 130 patients for lithiasis, in 66 for stenosis, in 14 for recurrent parotitis of childhood, in 8 for recurrent sialadenitis of unknown origin and in 3 for radio-iodine induced sialadenitis. Complications occurred in 11 of 272 sialendoscopies (4\%). Those were iatrogenic perforations, temporary lingual nerve paresthesia and swelling of the floor of the mouth. $53 \%$ of patients returned the questionnaire, for a total of 146 evaluable sialendoscopies. The majority of the responders indicated that sialendoscopy had improved their symptoms (83.6\%). Salivary glands could be preserved in $89 \%$ of the responder group. The 
highest percentage of patients reporting residual symptoms was found in the RPC group (81.3\%) and the lowest in the lithiasis group (16.2\%). Besides age, no statistical differences in demographic and pathological features between the responder and non-responder groups were found, supporting generalization of the responders' results to the entire cohort.

Conclusions: This study confirms the good long-term outcomes of sialendoscopic interventions in patients with chronic sialadenitis of different etiologies and a high rate of gland preservation.

\section{Keypoints:}

- Sialendoscopy is a minimally invasive, gland-preserving technique.

- We found low rates of complications and high success rates, hereby confirming that sialendoscopy is a safe and efficient technique for benign salivary diseases.

- The questionnaire showed that a vast majority of the patients reported an improvement of their symptoms after sialendoscopy.

- The highest success rates were found for sialolithiasis.

- After conservative measures, sialendoscopy should be the first choice of treatment. INTRODUCTION

Sialendoscopy is a minimally invasive technique, which offers direct intraluminal visualization of both the submandibular and the parotid glandular ducts. It offers diagnostic and therapeutic possibilities and can be performed under local or general anesthesia. This technique preserves the gland, using smaller or no incisions, improves glandular function and shortens the recovery period as compared to gland removing approaches. $^{1}$

Minimally invasive techniques are a trend seen in every field of surgery. ${ }^{2}$ Gundlach and Katz introduced flexible endoscopes into the salivary duct for the first time in the early '90s. ${ }^{1}$ Marchal was the first to introduce a more practical semirigid modular endoscope. ${ }^{3}$ Progressively, micro-instruments were developed allowing treatment, like the retrieval of stones with forceps or baskets, the dilatation of stenotic ducts, the removal of granulation tissue, and the treatment of radioiodine-induced sialadenitis and recurrent parotitis of childhood (RPC). ${ }^{4-6}$ Association with extracorporeal shock wave or intracorporeal pneumatic or laser lithotripsy now also enables us to remove stones larger than $4 \mathrm{~mm}$, which initially formed a restriction of the endoscopic approach. ${ }^{4}$

Studies have shown that salivary gland diseases have a significant impact on the patient's quality of life. ${ }^{7}$

Sialolithiasis is the major cause of salivary gland diseases, estimated to contribute to $50-66 \%$ of all observed problems. ${ }^{4}$ It is most common in the submandibular gland (80-95\%), followed by the parotid gland (5-20\%), and rarely occurs in the sublingual gland and the minor salivary glands $(2 \%){ }^{4,8}$ Salivary duct scarring is seen in $25 \%$, making it the second most common cause of obstructive disease. It is most common in the parotid gland $(75 \%) .^{2}$ In patients with obstructive gland disease, initial treatment should be conservative. ${ }^{4}$ If this approach fails, sialendoscopy should be considered rather than an open surgical approach. Success rates of therapeutic sialendoscopy range between $85 \%$ and $95 \%$, thus drastically reducing the need for removal of the gland and the risk of complications. ${ }^{4}$ The patient-perceived benefit and the improvement in quality of life has been demonstrated earlier. ${ }^{9-12}$ This study aims to evaluate the results and complications that were encountered during our 12-year experience with sialendoscopy at the $<$ Blinded for review $>$ department of Otorhinolaryngology, Head and Neck Surgery, to see whether the alleged advantages could also be confirmed in our cohort.

\section{PATIENTS AND METHODS}

\section{Patients}

This study is a retrospective cohort study with a prospective follow-up by questionnaires of all patients that underwent a sialendoscopy at the $<$ Blinded for review $>$ department of Otorhinolaryngology, Head and Neck Surgery between March 2008 and June 2020. 
Following an approval of the Research Ethics Committee (MP004975), the electronic files of 221 patients were searched for information about their sialendoscopic procedure and follow-up consultations. The same patients were also asked by mail to fill out a questionnaire. Non-responders where contacted again by phone and asked once more, if they agreed to fill out the questionnaire. Non-Belgian residents and patients who passed away were excluded.

\section{Procedure}

Sialendoscopies were performed using two types of endoscopes, the 'modular Marchal scope' (diagnostic 0.9 $\mathrm{mm} /$ therapeutic $1.6 \mathrm{~mm}$ ) and the 'Marchal all-in one scope' with diameters of $1.1 \mathrm{~mm}, 1.3 \mathrm{~mm}$ and 1.6 $\mathrm{mm}$ (Karl Storz, Tüttlingen, Germany). The most frequently used additional tools were a basket for stone retrieval, a guidewire for stenosis cases, and a Holmium-Yag laser $(10 \mathrm{~Hz}, 0,50 \mathrm{~J} /$ pulse $)$ for intraductal laser fragmentation of stones (Rocamed, Monaco). Dilatation of stenosis was practically done by introducing increasing diameters of sialendoscopes, starting from with the $0.9 \mathrm{~mm}$ diameter, preferably over an initially inserted guide wire. In the case of RPC and stenosis, an injection with $1 \mathrm{ml}$ long-acting corticosteroids (betamethason, acetate $3 \mathrm{mg} / \mathrm{ml}$; betamethason, sodiumphosphate $4 \mathrm{mg} / \mathrm{ml}$; Celestone ß, MSD, Kenilworth, USA) was performed. If sialendoscopy alone was not successful, a combined approach technique was performed. For parotid pathology, this could be a combined approach with an external parotidectomy incision approach or an intraoral semilunar incision anteriorly from the ostium of Stensens' duct. For submandibular pathology, an endoscope-guided incision was made in the back of the floor of the mouth to find the duct, while preserving the lingual nerve. ${ }^{13}$

\section{Methods}

From the electronic patient files, we extracted following variables: gender, age at first intervention, location of the symptomatic salivary gland, indication for sialendoscopy, findings at sialendoscopy, complications and outcome as assessed during follow-up consultations in our department. Peroperatively, we used the LSD classification ${ }^{14}$ (Table 1) to prospectively classify the different salivary gland pathologies that were encountered. To assess symptom control and gland-related quality of life at long-term follow-up, we used the questionnaire designed by Gillespie et $\mathrm{al}^{10}$.

\section{Statistical analysis}

SPSS Statistics 27.0 (IBM, Armonk, NY) was used to perform the statistical analysis. The Fisher exact test and Chi squared test were used to determine the statistical significance of differences for categorical variables, the Mann-Whitney U test was performed when numerical variables were involved, and the Mc Nemar test was used to compare paired groups. Statistical significance was set at $\mathrm{p}<0.05$.

\section{RESULTS}

Of the 221 contacted patients, initially only 101 returned the questionnaire (response rate $45.7 \%$ ). Patients who did not respond to the first attempt, were contacted again. This resulted in a rather disappointing 15 extra responders (final response rate $52.5 \%$ ). Of 116 patients who filled out the questionnaire, 15 underwent a bilateral intervention or had - during follow-up - undergone more than 1 sialendoscopic intervention. A questionnaire was filled out for each procedure that was performed. This resulted in 146 long-term evaluable sialendoscopies of the total of 272 that were carried out during the study period.

\section{General descriptive analysis}

Patients demographics and clinical information of the responders and non-responders are summarized in Table 2 and 3.

Lithiasis was found in $50.7 \%$ of the total population and most frequently seen in the submandibular gland (76.8\%). If lithiasis was found, retrieval of the stone, either by sialendoscopy alone $(n=45)$ or by combined approach $(\mathrm{n}=76)$, was successful in $90.9 \%$ of the total population. When comparing success rates between on the one hand the responder $(87.3 \%)$ and non-responder group $(94.8 \% ; \mathrm{p}=0.150)$ and on the other hand the parotid $(83.3 \%)$ and submandibular group $(92.8 \% ; \mathrm{p}=0.149)$ there were no significant differences (Chi-square 
test). In 10 patients no stone was found during the procedure, despite preoperative diagnosis of lithiasis. The chance of success of stone retrieval depended on the L classification, as summarized in Table 4. As expected, good results were seen in the L1 and L2a classification groups, but also the L3a classification group showed a high percentage of successful retrieval. All but two retrieval failures were with fixed stones (L2 or greater; $\mathrm{n}=81$ ).

Stenosis was the most frequent pathology in the parotid gland (57\%). Sialendoscopy was most frequently performed within a combined approach setting for lithiasis $(55.1 \%)$ than for addressing other pathologies (only $15.7 \%$ of non-lithiasis parotid cases needed a combined approach; $\mathrm{p}<0.01$, Chi-square test).

Complications occurred in 11 out of 272 sialendoscopies. Seven patients had iatrogenic perforations, 2 patients a temporary lingual nerve paresthesia following combined submandibular approach and 2 patients had a pronounced but temporary swelling of the floor of the mouth which did not necessitate additional treatment.

\section{Questionnaire}

The results of the questionnaire are summarized in Table 5 .

The median follow-up time between intervention and filling out of the questionnaire was 37 months (2-126 months). The majority of patients (64.4\%) reported to have no residual symptoms.

Patients with residual symptoms (35.6\%) were further evaluated. When they were asked to rate their current pain on a scale from 0 (no pain) to 10 (severe pain and need for pain relief), the median score was $3(0-9$, $\mathrm{SD}=2.5)$. When asked to rate their symptoms improvement, the median score was $7(0-9, \mathrm{SD}=3.1)$. In $31.5 \%$, the patient indicated that their salivary gland problem still had an impact on their quality of life, such as the need of carrying a water bottle or frequently using gums or candies to keep their mouth moist.

Eventual gland excision was only performed in 16 patients (11\% of total in the responder group), most frequently because of a non-retrievable stone, recurrence of a stone or recurrent sialadenitis. All gland excisions where performed in our department.

Neither caffeine consumption $(\mathrm{p}=0.440)$, smoking $(\mathrm{p}=0.171)$ nor xerostomia inducing medications $(\mathrm{p}=0.449)$ were, on univariate analysis (Chi-square test) significantly associated with a higher rate of residual symptoms (Of note, bilateral sialendoscopic interventions were significantly associated with a higher rate of residual symptoms $(\mathrm{p}<0.01$, Chi-square test $))$.

During the first postoperative checkup, $16.4 \%$ of the 136 responders (10 patients were lost to follow-up at first checkup) reported they still had residual symptoms. However, at the time they completed the questionnaire they reported residual symptoms in $35.6 \%$, which is significantly higher $(\mathrm{p}<0.01$, Mc Nemar test).

Parotid sialendoscopies were associated with a higher percentage of patients reporting residual symptoms (46.9\%) as compared to $26.8 \%$ of patients undergoing sialendoscopy of the submandibular gland $(\mathrm{p}=0.012$, Chi-square test). However, there was no significant difference between the parotid and submandibular group when scaling both their current pain and improvement on a scale from 0 to 10 (respectively $\mathrm{p}=0.597$ and $\mathrm{p}=0.566$, Mann-Whitney $\mathrm{U}$ test). Resection of the parotid gland was necessary in $9.4 \%$ and of the submandibular gland in $12.2 \%$ in the responder group ( $\mathrm{p}=0.588$, Chi-square test).

The highest percentage of patients reporting residual symptoms was found in the RPC group (81.3\%). For the pathology groups "stenosis", "recurrent sialadenitis" and "lithiasis" this was respectively $44.6 \%, 50 \%$ and $16.2 \%$, a trend to significant difference ( $\mathrm{p}<0.01$, Chi-square test). For the RPC group however, all of the patients indicated that sialendoscopy improved their symptoms. The median score when asked to rate both their pain and improvement on a scale from 0 to 10 was respectively 3 and 8 . Preservation of the gland was possible in $85.3 \%$ in the lithiasis group, $89.3 \%$ in the stenosis group and $100 \%$ in the RPC and recurrent sialadenitis group

\section{DISCUSSION}


This study describes the population characteristics and the results of all sialendoscopies (272) that were performed in our department between March 2008 until and June 2020. Furthermore, we evaluated the long-term outcome for the 146 sialendoscopies performed in 116 patients who returned their questionnaire. Median time between the intervention and completing the questionnaire was 37 months.

Regarding the total of 272 sialendoscopies performed, stone removal was successful in 111 out of 138 lithiasis patients $(80.4 \%)$. This is in the range of successful endoscopic stone removal in the literature (70-90\%). ${ }^{2}$ All but two retrieval failures were in fixed stones (L2 or greater; stones larger than the ductal diameter). Indeed, in our cohort, in line with the literature, L2b and L3b classification stones implied lower rates of successful retrieval and higher rates of recurrence. ${ }^{15-18}$ The mean diameter of the submandibular duct is $1.5 \mathrm{~mm}$ and the diameter of the parotid duct is 1.2 to $1.4 \mathrm{~mm} .{ }^{13}$ When there is a stone, usually the duct gets dilated proximally of the obstruction. A stone that moves more proximally in the duct, can become a "floating stone", even if the stone itself is e.g. 2 or $3 \mathrm{~mm}$ in diameter. Those are the stones that can be trapped in a basket and pulled toward the distally located, smaller diameter. It is at this point that an incision needs to be made to deliver the stone. Preferably this is at the level of the papilla, but if the basket-trapped stone gets stuck more proximally, a floor of mouth incision for submandibular stones, and a semilunar incision in the cheek or an external approach to the parotid may be needed. This concept explains that stones up to 4 $\mathrm{mm}$ in the submandibular duct and up to $3 \mathrm{~mm}$ in the parotid duct, (measured perpendicular to the duct) can be successfully extracted with baskets, as reported by Marchal et al ${ }^{17}$ and confirmed by Walvekar et al. ${ }^{18}$ For larger stones, fragmentation of the stone is necessary before basket retrieval becomes an option. ${ }^{17}$

We observed that, in the majority of patients in the lithiasis group (55.1\%), sialendoscopy alone was not sufficient, and a combined approach was needed to retrieve the stone. In non-lithiasis pathologies, a combined approach was needed in only $15.7 \%(\mathrm{p}<0.01)$. Gillespie et $\mathrm{al}^{10}$ found similar results, with, likewise, a combined approach rate needed in the majority of patients in their lithiasis group (83\%). Conversely, the majority of their patients with non-lithiasis pathology $(77 \%)$ could be helped by sialendoscopy alone, and we found similar results $(82.8 \%){ }^{10}$

Regarding the results of the questionnaire, symptom improvement was found in $83.6 \%$ and salivary glands could be preserved in $89 \%$. This is comparable with results described in the literature. The study of Gillespie et $\mathrm{al}^{10}$ reported symptom improvement in $89 \%$ and retention of the salivary gland in $92 \%$.

Another study on 1,154 patients with lithiasis reported that $82 \%$ of them had no residual symptoms and salivary glands could be preserved in $96 \% .{ }^{19}$ These results are similar to our findings in the lithiasis subgroup where we found no residual symptoms in $83.8 \%$ and preservation of the salivary gland in $85.3 \%$. A systematic review showed success rates of $86 \%$ for sialendoscopy alone and $93 \%$ when combined with a minimally invasive surgical approach in case of obstructive disease. ${ }^{20}$

In case of stenosis and RPC, the rates of residual complaints were higher with respectively $44.6 \%$ and $81.3 \%$. However, patients in the RPC group all reported an improvement of their symptoms with a median score of 8 on a scale from 0 to 10 . When comparing these results with the literature, we see a higher rate of recurrence compared to previous studies on the effectiveness of sialendoscopy in RPC cases. The systematic review of Garavello et $\mathrm{al}^{21}$ reported a common rate of recurrence of only $25.8 \%$, however none of the 19 studies were randomized, and most studies were case series or case reports with short follow-up. Even more, the definitions used for RPC varied widely, with some studies including children with only one or two episodes, causing an overestimation of the real success rate. We only proceeded to sialendoscopy in children with at least 6 consecutive episodes with intervals of less than 2 months. A small recent study on 33 patients with non-lithiasis related sialadenitis showed that only $59.3 \%$ was symptom-free after sialendoscopy. ${ }^{22}$ As previously reported by Gillespie et $\mathrm{al}^{10}$, the higher rate of clearance of symptoms in the lithiasis group can be explained by the impression that an obstruction that can be removed is more likely to lead to a better outcome compared to an obstruction due to inflammation or scar tissue, that can be improved but not completely resolved. ${ }^{10}$ This conclusion was also made by Aubin-Pouliot et al ${ }^{9}$ who found better outcomes for the lithiasis group as well, as compared to non-lithiasis related obstructive disease. 
Sialendoscopies of the parotid gland were associated with a significantly higher percentage of residual symptoms compared to the submandibular gland $(\mathrm{p}=0.012)$. This can probably be explained by the fact that stenosis and RPC was more often observed in the parotid gland than the prognostically better lithiasis-related pathologies.

Like also observed by Gillespie et $\mathrm{al}^{10}$, we found that bilateral sialendoscopic interventions were associated with a significant higher rate of residual symptoms on univariate analysis $(\mathrm{p}<0.01)$. This is most likely due to the underlying pathology (inflammatory causes of chronic sialadenitis), which is related to a worse outcome. ${ }^{10}$

When we compared the recurrence of symptoms at the first post-operative check-up and the long-term followup with the questionnaire, we notice a progressive and significant $(\mathrm{p}<0.01)$ reappearance of complaints at long-term follow-up. This suggests that being initially symptom-free does not necessarily predict a favorable long-term outcome. Gillespie et $\mathrm{al}^{10}$ compared follow-up time for less than their median 17 months with a time greater than 17 months and did not notice a difference in symptom deterioration with longer follow-up time. However, they did not compare the long-term outcome with the first post-operative results, like we did in this study.

When comparing demographic features, pathology distribution and early postoperative results among the responders and non-responders, only for age, a statistical difference was found. Non-responders were significantly younger than responders; an observation that we cannot explain. Nevertheless, given the absence of other differences between the two groups, we believe it is fair to say that the results of the questionnaire are probably representative for the entire patient group.

In general, as compared to external gland removing surgery, sialendoscopy is a safe procedure. In our study complications occurred in only 11 out of the 272 procedures (4\%). Nahlieli et al $^{23}$ found a $3.23 \%$ complication rate in a study with 526 patients, treated with interventional sialendoscopy or endoscopy assisted intraoral extraductal interventions. The complications they found were strictures (1.7\%), traumatic ranulas (1.7\%) and lingual nerve paresthesia $(0.72 \%){ }^{23}$ The rate of complications after traditional surgery is higher. Following parotid or submandibular gland removal, there is a scar and contour deformity. On top of that, according to Capaccio et $\mathrm{al}^{24}$, the most important complications after parotidectomy are temporary $(2 \%-76 \%)$ and permanent facial nerve injury (1\%-3\%), sensory damage resulting from the greater auricular nerve $(2 \%$ $100 \%)$ and Frey syndrome (8\%-33\%). After removal of the submandibular gland, they reported permanent marginal mandibular nerve injury (1\%-8\%), hypoglossal nerve injury (3\%), and lingual nerve injury (2\%). ${ }^{24}$

The limitations of this study are related to its retrospective nature and the suboptimal response rate. For example, Gillespie et $\mathrm{al}^{10}$ obtained survey data in $67 \%$, compared to $52.5 \%$ in our study. Another similar study previously done, showed a lower response rate than the current study $(43 \%) .{ }^{9}$ The suboptimal response rate is due to the long follow-up time (about 5 years longer than in the study of Gillespie et $\mathrm{al}^{10}$ ) and therefore difficulties for the patient to recall the intervention (recall bias) and the impossibility to reach patients who were treated several years ago (information bias). This can lead to an over- or underrepresentation of dissatisfied patients. This study doesn't address the issue of cost-effectiveness of sialendoscopy. In times of savings in health care this aspect must be studied in the future. ${ }^{32}$

\section{CONCLUSION}

This study confirms that sialendoscopy is a safe and efficient, gland saving technique for benign salivary diseases. Therefore, it should be the first choice of treatment after conservative measures. The vast majority of the patients indicated that sialendoscopy improved their symptoms. Success rates were highest for the lithiasis subgroup. The questionnaire designed by Gillespie et $\mathrm{al}^{10}$ showed to be a good tool for measurement of the satisfaction of patients after sialendoscopy and the improvement of their salivary gland pathology.

\section{CONFLICT OF INTEREST}

This study received no funding. There is no potential or actual conflict of interest with respect to this study. 


\section{DATA AVAILABILITY STATEMENT}

The data that support the findings of this study are available from the corresponding author upon reasonable request.

\section{References}

1. Katz P. New method of examination of the salivary glands: the fiberscope. Inf Dent .1990; 72: 785-6

2. Erkul E, Cekin E, Gungor A. Long-Term Outcomes of Sialendoscopy in the management of Sialolithiasis and Idiopathic Chronic Sialadenitis with Ductal Scars. Turk Arch Otorhinolaryngol. 2019; 57(2): 7580 .

3. Marchal F, Dulguerov P, Lehmann W. Interventional sialendoscopy. N Engl J Med 1999; 341:1242-1243

4. Carta F, Farneti P, Cantore S, Macri G, Chuchueva N, Cuffaro L, et al. Sialendoscopy for Salivary Stones: Principles, Technical Skills and Therapeutic Experience. Acta Otorhinoaryngologica Italica. 2017; 37(2): 102-112. https://doi.org/10.14639/0392-100X-1599

5. Bomeli SR, Schaitkin B, Carrau RL, Walvekar RR. Interventional Sialendoscopy for Treatment of Radioiodine-Induced Sialadenitis. The Laryngoscope. 2009;119 (5): 864-67. https://doi.org/10.1002/lary.20140.

6. Ramakrishna J, Strychowsky J, Gupta M, Sommer DD. Sialendoscopy for the Management of Juvenile Recurrent Parotitis: A Systematic Review and Meta-Analysis: Sialendoscopy for the Management of JRP. The Laryngoscope. 2015; 125(6): 1472-79. https://doi.org/10.1002/lary.25029.

7. Koch M, Iro H. Salivary Duct Stenosis: Diagnosis and Treatment. Acta Otorhinolaryngologica Italica. 2017 Apr; 37(2): 132-141. https://doi.org/10.14639/0392-100X-1603.

8. Meulemans J, Hens G, Vander Poorten V. Current management of obstructive sialolithiasis. Journal of ENT Masterclass. 2012; 5:55-69

9. Aubin-Pouliot A, Elise AD, David WE, Chang JL, Ryan WR. The Chronic Obstructive Sialadenitis Symptoms Questionnaire to Assess Sialendoscopy-Assisted Surgery: Assessing Sialendoscopy With the COSS Questionnaire. The Laryngoscope. 2016;126, (1): 93-99. https://doi.org/10.1002/lary.25521.

10. Gillespie MB, O'Connell BP, Rawl JW, McLaughlin CW, Carroll WW, Nguyen SA. Clinical and Quality-of-Life Outcomes Following Gland-Preserving Surgery for Chronic Sialadenitis: QOL After Surgery for Chronic Sialadenitis. The Laryngoscope. 2015;125, (6) :1340-44. https://doi.org/10.1002/lary.25062.

11. Koch Ml, Kunzel J, Iro H, Psychogios G, Zenk J. Long-Term Results and Subjective Outcome after Gland-Preserving Treatment in Parotid Duct Stenosis: Parotid Stenosis Long-Term Results. The Laryngoscope. 2014;124, (8): 1813-18. https://doi.org/10.1002/lary.24534.

12. Meier BA, Holst R, Lars PS. Patient-Perceived Benefit of Sialendoscopy as Measured by the Glasgow Benefit Inventory: Patient-Perceived Benefit of Sialendoscopy. The Laryngoscope. 2015;125 (8) : 1874-78. https://doi.org/10.1002/lary.25369.

13. Marchal F. SIALENDOSCOPY: The Hands-On-Book. Annecy, France: Artcast Medical. 2015. 351p.

14. Marchal F, Chossegros C, Faure F, Delas B, Bizeau A, Mortensen B, et al. Salivary stones and stenosis: A comprehensive classification. Rev Stomatol Chir Maxillofac. 2008; 109:233-236. https://doi.org/10.1016/j.stomax.2008.07.004.

15. Cox D, Chan L, Veivers D. Prognostic Factors for Therapeutic Sialendoscopy. The Journal of Laryngology \& Otology. 2018;132(03):275-78. https://doi.org/10.1017/S0022215117000822.

16. Foletti JM, Graillon N, Avignon S, Guyot L, Chossegros C. Salivary Calculi Removal by Minimally Invasive Techniques: A Decision Based Tree Based on the Diameter of the Calculi and Their Position in the Excretory Duct. J Oral Maxillofac Surg. 2018 ; 76: 112-118. https://dx.doi.org/10.1016/j.joms.2017.06.009. 
17. Marchal F, Dulguerov P. Sialolithiasis management: the state of the art. Arch Otolaryngol Head Neck Surg. 2003;129(9):951-6

18. Walvekar RR, Carrau RL, Schaitkin B. Endoscopic sialolith removal: orientation and shape as predictors of success. Am J Otolaryngol. 2009;30(3):153-6. doi: 10.1016/j.amjoto.2008.03.007.

19. Zenk J, Koch M, Klintworth N, et al. Sialendoscopy in the diagnosis and treatment of sialolithiasis: a study on more than 1000 patients. Otolaryngol Head Neck Surg 2012; 147: 858-863

20. Strychowsky JE, Sommer DD, Gupta MK, Cohen N, Nahlieli O. Sialendoscopy for the Management of Obstructive Salivary Gland Disease A Systematic Review and Meta-analysis. Arch Otolaryngol Head Neck Surg. 2012;138(6): 541-547.doi:10.1001/archoto.2012.856

21. Garavello W, Redaelli M, Galluzzi F, Pignataro L. Juvenile recurrent parotitis: A systematic review of treatment studies. International Journal of Pediatric Otorhinolaryngology. 2018;112(09); 151-157. https://doi.org/10.1016/j.ijporl.2018.07.002

22. Donovan E, Kwok SL, Woei SL. Efficacy of Sialendoscopy in the Management of Noncalculi-Related Sialadenitis. J Oral Maxillofac Surg. 2020. https://doi.org/10.1016/j.joms.2020.01.021

23. Nahlieli O. Complications of Sialendoscopy: Personal Experience, Literature Analysis, and Suggestions. Journal of Oral and Maxillofacial Surgery. 2015;73(1): 75-80. https://doi.org/10.1016/j.joms.2014.07.028

24. Capaccio P, Torretta S, Pignataro L. The role of adenectomy for salivary gland obstructions in the era of sialendoscopy and lithotripsy. Otolaryngol Clin North Am. 2009;42(6):1161-71

25. Jokela J, Saarinen R, Makitie A, Sintonen H, Roine R. Costs of sialendoscopy and impact on health-related quality of life. European Archives of Oto-Rhino-Laryngology. 2019; 276:233-241. https://doi.org/10.1007/s00405-018-5196-9. 